\title{
The ASTRI mini-array within the future Cherenkov Telescope Array
}

\author{
Stefano Vercellone ${ }^{1, \mathrm{a}}$ for The ASTRI Collaboration ${ }^{2}$, The CTA Consortium ${ }^{3}$ \\ ${ }^{1}$ INAF/IASF Palermo, via U. La Malfa 153, 90146 Palermo, Italy \\ 2 http://www.brera.inaf.it/astri/ \\ ${ }^{3}$ https://portal.cta-observatory.org
}

\begin{abstract}
The Cherenkov Telescope Array (CTA) is a large collaborative effort aimed at the design and operation of an observatory dedicated to very high-energy gamma-ray astrophysics in the energy range from a few tens of $\mathrm{GeV}$ to above $100 \mathrm{TeV}$, which will yield about an order of magnitude improvement in sensitivity with respect to the current major arrays (H.E.S.S., MAGIC, and VERITAS). Within this framework, the Italian National Institute for Astrophysics is leading the ASTRI project, whose main goals are the design and installation on Mt. Etna (Sicily) of an end-to-end dual-mirror prototype of the CTA small size telescope (SST) and the installation at the CTA Southern site of a dual-mirror SST miniarray composed of nine units with a relative distance of about $300 \mathrm{~m}$. The innovative dual-mirror Schwarzschild-Couder optical solution adopted for the ASTRI Project allows us to substantially reduce the telescope platescale and, therefore, to adopt silicon photo-multipliers as light detectors. The ASTRI mini-array is a wider international effort. The mini-array, sensitive in the energy range 1-100 $\mathrm{TeV}$ and beyond with an angular resolution of a few arcmin and an energy resolution of about $10-15 \%$, is well suited to study relatively bright sources (a few $\times 10^{-12} \mathrm{erg} \mathrm{cm}^{-2} \mathrm{~s}^{-1}$ at $10 \mathrm{TeV}$ ) at very high energy. Prominent sources such as extreme blazars, nearby wellknown BL Lac objects, Galactic pulsar wind nebulae, supernovae remnants, micro-quasars, and the Galactic Center can be observed in a previously unexplored energy range. The ASTRI mini-array will extend the current IACTs sensitivity well above a few tens of $\mathrm{TeV}$ and, at the same time, will allow us to compare our results on a few selected targets with those of current (HAWC) and future high-altitude extensive air-shower detectors.
\end{abstract}

\section{Introduction}

The very high-energy (VHE) portion of the electromagnetic spectrum (above $\approx 100 \mathrm{GeV}$ ) is currently being investigated by means of ground-based imaging atmospheric Cherenkov telescopes (IACTs, see [1] for a recent review). In order to dramatically boost the current IACT performance and to widen the VHE science, a new Cherenkov telescope array (CTA) has been proposed, as described in [2] and more recently in [3]. The wide energy range covered by the CTA (from a few tens of $\mathrm{GeV}$ to above $100 \mathrm{TeV}$ ) requires different kinds of telescopes. Four large size telescopes (LSTs, D $23 \mathrm{~m}$ ) will be placed at the center of the array, to lower the energy threshold down to a few tens of GeV. A few tens of medium size telescopes (MSTs, D 12 m, SCTs, D 9.5 m) will cover approximately $1 \mathrm{~km}^{2}$, to improve by

\footnotetext{
a e-mail: stefano.vercellone@iasf-palermo.inaf.it
} 

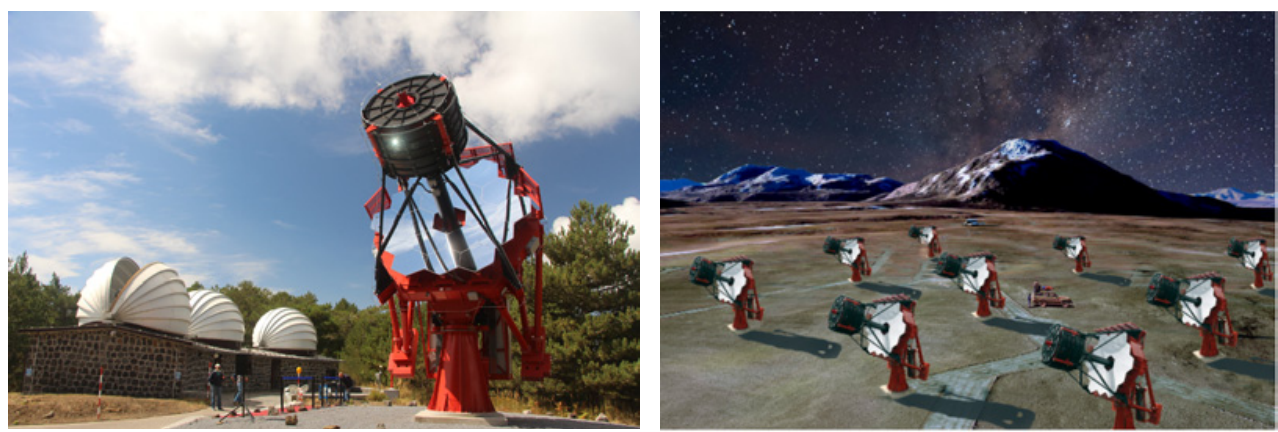

Figure 1. Left panel: the ASTRI SST-2M prototype at the Serra La Nave observing station. Right panel: Artistic concept (not to scale) of the ASTRI mini-array.

a factor of ten the sensitivity in the energy range $0.1-10 \mathrm{TeV}$. Finally, 70 small size telescopes (SSTs, primary mirror D $4 \mathrm{~m}$, Aeff $\sim 5-10 \mathrm{~m}^{2}$ ) covering about $10 \mathrm{~km}^{2}$ will enhance Galactic plane source studies in the energy range beyond $100 \mathrm{TeV}$. A detailed review of the CTA project is given in [4].

\section{The ASTRI Project and its end-to-end prototype}

Within the CTA framework, the Italian National Institute for Astrophysics (INAF) is leading the "Astrofisica con Specchi a Tecnologia Replicante Italiana" (ASTRI) Flagship Project [5] of the Ministry of Education, University and Research. Primarily, INAF has designed and developed an end-to-end prototype of the CTA small-size telescope in a dual-mirror configuration (SST-2M). This prototype is currently being tested under field conditions at the INAF "M.C. Fracastoro" observing station in Serra La Nave (Mount Etna, Sicily). The ASTRI SST-2M prototype was recently inaugurated during the CTA Consortium Meeting in September 2014. Figure 1 (left panel) shows the prototype in front of the domes. Currently all telescope sub-systems are in place and properly working. The camera is being assembled, an it will be installed at the telescope focal plane at the beginning of 2016.

A new dual-mirror, Schwarzchild-Couder (SC) aplanatic design has been proposed [6]. In the SC telescope, the focal plane is located in-between two aspherical mirrors, close to the secondary mirror. No Cherenkov telescope has adopted this optical system before. The dual-mirror optical system will reduce the dimension, the weight, and the cost of the camera at the focal plane of the telescope, and will obtain a more compact and stiffer mechanical structure, and an optimal imaging resolution across a wide field of view. Moreover, due to the reduced plate-scale, silicon-based photo-multipliers (SiPMs) can be adopted as light detectors.

The ASTRI SST-2M prototype adopts a segmented $4.3 \mathrm{~m}$ primary mirror (M1) composed of 18 facets, a monolithic $1.8 \mathrm{~m}$ secondary mirror $(\mathrm{M} 2$, with a radius of curvature of $2.2 \mathrm{~m}$ ), a focal length $\mathrm{F}=2.15 \mathrm{~m}$, a field of view $\mathrm{FoV} \sim 9.6^{\circ}$, for a ratio $\mathrm{F} / \mathrm{D}_{1}=0.5$. The mirror manufacturing process is the "glass cold shaping" technique, specifically developed by INAF for Cherenkov mirrors $[7,8]$. The curved focal plane ( $\sim 1 \mathrm{~m}$ of radius of curvature) hosts 1984 logical pixels $\left(6.2 \mathrm{~mm} \times 6.2 \mathrm{~mm}, 0.17^{\circ}\right)$. The current photo-sensors are Hamamatsu S118283344M silicon-based photo-multipliers, but other sensors are also being tested [9]. The ASTRI camera [10] is extremely compact $(\sim 50 \mathrm{~cm} \times 50 \mathrm{~cm} \times 50 \mathrm{~cm})$ and light $(\sim 50 \mathrm{~kg})$. Contrary to other CTA telescopes adopting a signal-sampler front-end electronics (FEE), the 


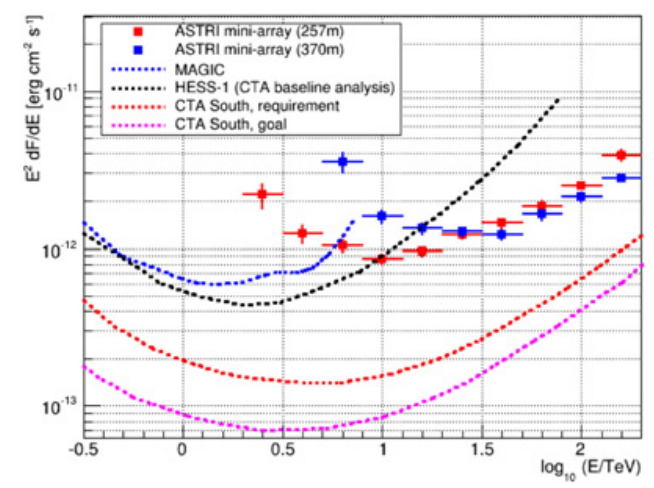

Figure 2. ASTRI mini-array differential sensitivity for 9 telescopes (blue and red points, for two different telescope relative distances) compared to the MAGIC, H.E.S.S.-I, and CTA (requirements and goal) ones (blue, black, red, and magenta dashed lines, respectively). See [13] and references therein.

ASTRI camera adopts as FEE the CITIROC, a customized version of the EASIROC [11] ASIC signal-shaper manufactured by Omega ${ }^{1}$.

We are completing the commissioning phase during which we tested all the mechanical and optical subsystems, as well as the control software. The commissioning of the camera will take place in Spring 2016. A science verification phase, during which we will observe Mrk 421, Mrk 501, and afterwards the Crab Nebula, will start at the end of Spring 2016. The results of these observations will allow us to cross-check the prototype performance with our Monte Carlo simulations. We estimate that, in the sensitivity range $(\mathrm{E} \geq 2 \mathrm{TeV})$, we can detect a flux level of $1 \mathrm{Crab}$ at $5 \sigma$ in a few hours [12].

\section{The ASTRI mini-array}

A remarkable improvement in terms of performance could come from the operation, in late 2016, of a mini-array, composed of nine SST-2M telescopes (see Fig. 1, right panel, for an artistic concept) to be placed at the final CTA southern site. The ASTRI miniarray is a collaborative effort led by INAF in synergy with the University of São Paulo and FAPESP (Brazil) and the North-West University (South-Africa). Preliminary Monte Carlo simulations [13] yield an improvement in sensitivity that, for nine telescopes, could surpass the H.E.S.S. sensitivity above $10 \mathrm{TeV}$, extending it up to about $100 \mathrm{TeV}$, as shown in Fig. 2. The ASTRI mini-array will be able to study in detail relatively bright (a few $\times 10^{-12} \mathrm{erg} \mathrm{cm}^{-2} \mathrm{~s}^{-1}$ at $10 \mathrm{TeV}$ ) sources with an angular resolution of a few arcmin and an energy resolution of about $10-15 \%$.

Thanks to the array approach, it will be possible to verify the wide FoV performance to detect very high energy showers with the core located at a distance up to $500 \mathrm{~m}$ and to compare the mini-array performance with the Monte Carlo expectations by means of deep observations of a few selected targets. Moreover, it will be possible to perform the first CTA science, with its first solid detections during the first year of operation, as described in [14]. Prominent sources such as extreme blazars (KUV 00311-1938), nearby wellknown BL Lac objects (Mrk 501) and radio-galaxies (M 87), galactic pulsar wind nebulae (Crab Nebula, Vela-X), supernovae remnants (Vela-junior, RX J1713.7-3946), as well as

\footnotetext{
${ }^{1}$ http: //omega.in2p3.fr/; manufactured under INAF intellectual property.
} 

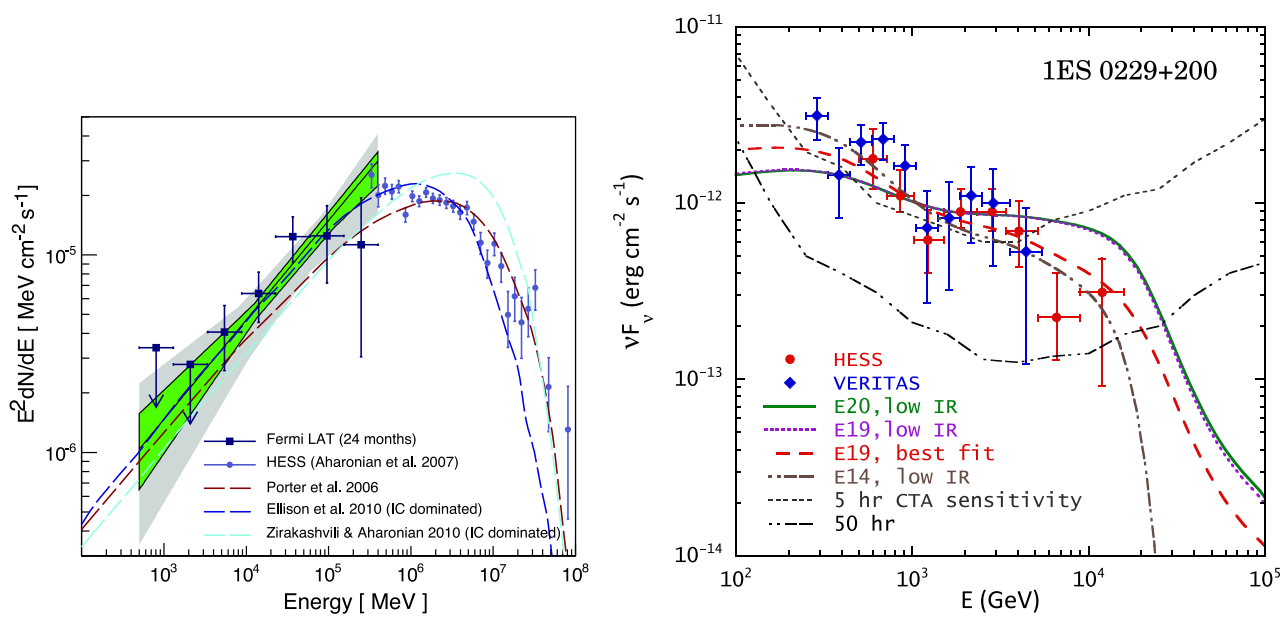

Figure 3. Left panel: supernova remnant RX J1713.7-3946 (see [15] for details). We expect for the ASTRI mini-array sensitivity at least comparable to (or slightly better than) the H.E.S.S. one above a few TeV. Right panel: spectral energy distribution of the extreme blazar 1ES 0229+200 (see [16] for details).

the Galactic Center can be observed in a previously unexplored energy range, in order to investigate the electron acceleration and cooling, relativistic and non relativistic shocks, the search for cosmic-ray (CR) PeVatrons, the study of the CR propagation, and the impact of the extragalactic background light on the spectra of the sources.

On the Galactic plane, one of the best targets is RX J1713.7-3946. Its detection by Fermi [15] and the combined study with H.E.S.S. (Fig. 3, left panel) shows that the highenergy and very high-energy (VHE) emission could be interpreted in the framework of a leptonic scenario. The improved and uniform sensitivity (within a few degrees off-axis) and the comparable angular resolution of the ASTRI mini-array at $\mathrm{E} \geq 10 \mathrm{TeV}$ with respect to the current IACTs could allow us to investigate the VHE emission in the different regions of this source, studying their spectra, and to extend the current spectral energy distribution (SED) well above a few tens of $\mathrm{TeV}$, searching for possible spectral cut-offs. Figure 3 (right panel) shows the SED of the extreme blazar 1ES 0229+200 with superimposed different theoretical SED fits (both hadronic and leptonic) assuming different EBL models (see [16] for a detailed discussion) and the 5- $\sigma$ differential sensitivity for 5 and $50 \mathrm{hr}$ observations with CTA (configuration E, as reported in [2]). Because of the uncertainty in EBL models, it is not easy to distinguish between the hadronic and leptonic scenarios at $\sim 1-10 \mathrm{TeV}$ energies. At higher energies, however, UHECR-induced cascade emission becomes harder than the gamma-ray one. A detection of $\geq 25 \mathrm{TeV}$ gamma-rays from 1ES $0229+200$ would only be consistent with an hadronic scenario.

\section{Synergies and conclusions}

The ASTRI mini-array will operate when the other IACTs will still be active. Compared to them, the ASTRI mini-array will extend the sensitivity up to $100 \mathrm{TeV}$ and beyond, a neverexplored energy range by IACTs. Moreover, it will benefit from a much larger field of view which will allow us to study in detail extended sources at energies about a decade higher than what is currently being explored and to monitor simultaneously a few close-by sources 
during the same pointing. Long exposures will be preferred, restricting the number of possible targets, and extending the observations also during moon light periods, thanks to the use of a SiPMs-based camera.

The lower imaging energy threshold of current and future extended air-shower (EAS) detectors $(\sim 100 \mathrm{GeV})$ and the wider energy range of the ASTRI mini-array (beyond $100 \mathrm{TeV}$ ) will allow us a direct comparison of scientific data (spectra, light-curves, integral fluxes) of those sources which could be monitored simultaneously, although on different integration time-scales. Moreover, the high-energy boundary of both EAS and the ASTRI mini-array will allow us to study the VHE $(\mathrm{E} \geq 10 \mathrm{TeV})$ emission from extended source such as SNRs and PWN, and to investigate the presence of spectral cut-offs. In summary, the ASTRI miniarray could be considered as the first CTA seed, allowing the entire CTA Consortium to start seminal studies on both Galactic and extra-galactic sources.

This work was partially supported by the ASTRI "Flagship Project" financed by the Italian Ministry of Education, University, and Research (MIUR) and led by the Italian National Institute of Astrophysics (INAF). We acknowledge partial support by the MIUR Bando PRIN 2009 and TeChe.it 2014 Special Grants. We also acknowledge support from the Brazilian Funding Agency FAPESP (Grant 2013/10559-5) and from the South African Department of Science and Technology through Funding Agreement 0227/2014 for the South African Gamma-Ray Astronomy Programme. We gratefully acknowledge support from the agencies and organizations listed in this page: http://www. cta-observatory.org/?q=node/22.

\section{References}

[1] J.A. Hinton, W. Hofmann, Annual Review of Astronomy \& Astrophysics 47, 523 (2009)

[2] M. Actis, G. Agnetta, F. Aharonian, A. Akhperjanian, J. Aleksić, E. Aliu, D. Allan, I. Allekotte, F. Antico, L.A. Antonelli et al., Experimental Astronomy 32, 193 (2011)

[3] B.S. Acharya, M. Actis, T. Aghajani, G. Agnetta, J. Aguilar, F. Aharonian, M. Ajello, A. Akhperjanian, M. Alcubierre, J. Aleksić et al., Astroparticle Physics 43, 3 (2013)

[4] J. Carr, This Volume (in press)

[5] G. Pareschi, the ASTRI Collaboration, the GATE Collaboration, the CHEC Collaboration, the CTA Consortium, Proc. 33rd ICRC (2013)

[6] V. Vassiliev, S. Fegan, P. Brousseau, Astroparticle Physics 28, 10 (2007)

[7] R. Canestrari, G. Bonnoli, E. Cascone, P. Conconi, G. Crimi, M. Fiorini, E. Giro, N. La Palombara, G. Pareschi, L. Perri et al., The ASTRI SST-2M prototype for the CTA: manufacturing of the structure and the mirrors, in SPIE Conference Series (2014), Vol. 9145

[8] R. Canestrari, G. Bonnoli, G. Crimi, M. Fiorini, E. Giro, N. La Palombara, G. Pareschi, L. Perri, G. Rodeghiero, G. Sironi et al., The glass cold-shaping technology for the mirrors of the CTA, in SPIE Conference Series (2014), Vol. 9151

[9] G. Bonanno, D. Marano, M. Belluso, S. Billotta, A. Grillo, S. Garozzo, G. Romeo, M. Timpanaro, Sensors Journal, IEEE 14, 3567 (2014)

[10] O. Catalano, M.C. Maccarone, C. Gargano, G. La Rosa, A. Segreto, G. Sottile, V. De Caprio, F. Russo, M. Capalbi, P. Sangiorgi et al., The camera of the ASTRI SST$2 M$ prototype for the Cherenkov Telescope Array, in SPIE Conference Series (2014), Vol. 9147

[11] S. Callier, C.D. Taille, G. Martin-Chassard, L. Raux, Physics Procedia 37, 1569 (2012), proceedings of the 2nd International TIPP Conference

[12] C. Bigongiari, the ASTRI Collaboration, Proc. 33rd ICRC (2013), arXiv: 1307.5006 
[13] F. Di Pierro, C. Bigongiari, A. Stamerra, P. Vallania, the ASTRI Collaboration, the CTA Consortium, Proc. TAUP 2015 (in press)

[14] S. Vercellone, the ASTRI Collaboration, Proc. 33rd ICRC (2013), arXiv:1307.5671

[15] A.A. Abdo, M. Ackermann, M. Ajello, A. Allafort, L. Baldini, J. Ballet, G. Barbiellini, M.G. Baring, D. Bastieri, R. Bellazzini et al., Astrophysical Journal 734, 28 (2011)

[16] K. Murase, C.D. Dermer, H. Takami, G. Migliori, Astrophysical Journal 749, 63 (2012) 